\title{
The logopenic variant of primary progressive aphasia
}

\author{
M.L. Henry and M.L. Gorno-Tempini \\ Memory and Aging Center, Department of Neurology, University of California, San Francisco
}

\begin{abstract}
Purpose-The aim of this review is to explore the evolution of the logopenic variant of primary progressive aphasia as a distinct clinical entity and to outline recent advances that have clarified its clinical characteristics, neural underpinnings and potential genetic and pathological bases. This is particularly relevant as researchers attempt to identify clinico-pathological relationships in subtypes of primary progressive aphasia in hopes of utilizing language phenotype as a marker of underlying disease.
\end{abstract}

Recent findings-Recent work has served to refine and expand upon the clinical phenotype of the logopenic variant. Logopenic patients show a unique pattern of spared and impaired language processes that reliably distinguish this syndrome from other variants of progressive aphasia. Specifically, they exhibit deficits in naming and repetition in the context of spared semantic, syntactic and motor speech abilities. Further, there is a growing body of evidence indicating a possible link between the logopenic variant phenotype and specific pathological and genetic correlates.

Summary-Findings indicate that the logopenic variant is a distinct subtype of progressive aphasia that may hold value as a predictor of underlying pathology. Additional research, however, is warranted in order to further clarify the cognitive-linguistic profile and to confirm its relation to certain pathological and genetic processes.

\section{Keywords}

logopenic progressive aphasia; primary progressive aphasia; logopenic variant; Alzheimer's disease

\section{Introduction}

The logopenic variant (also referred to as logopenic progressive aphasia or LPA) represents the most recently identified of the variants of primary progressive aphasia (PPA). The disorder is characterized by a unique language profile, caused by damage to specific anatomical areas, which in turn might have different probabilities of being associated with specific pathological or genetic processes. There is a growing body of research dedicated to clarifying the cognitive-linguistic, anatomical and pathological features of the logopenic variant. The purpose of this review is to outline its evolution as a clinical syndrome and to highlight recent advances that have helped to clarify the nature of the disorder.

Corresponding author: Maya L. Henry, Ph.D, Memory and Aging Center, Department of Neurology, University of California, San Francisco, mhenry@memory.ucsf.edu, (415)476-2912 .

This is a PDF file of an unedited manuscript that has been accepted for publication. As a service to our customers we are providing this early version of the manuscript. The manuscript will undergo copyediting, typesetting, and review of the resulting proof before it is published in its final citable form. Please note that during the production process errors may be discovered which could affect the content, and all legal disclaimers that apply to the journal pertain. 


\section{Primary progressive aphasia classification and the emergence of a third variant}

PPA is characterized as a slowly progressing, relatively isolated impairment of language, which results from neurodegenerative disease [1]. The disorder was first described in detail in the modern literature by Mesulam [2] and since that time has been the subject of a great deal of research aimed at clarifying its clinical phenotypes and neural and pathological bases. Whereas early reports often described PPA syndromes as being akin to aphasias resulting from stroke (e.g., Broca's and Wernicke's aphasia), it was soon recognized that the clinical presentation in PPA was both heterogeneous and distinct from vascular aphasias. Soon thereafter, efforts were made to classify PPA into clinical subtypes or variants. For more than twenty years, PPA patients were grouped broadly into two major types: nonfluent and fluent PPA. It became clear, however, that this binary classification scheme did not capture all patients adequately [3] and a third variant was occasionally mentioned in the literature [2,4]. Subsequently, Gorno-Tempini et al. [5] described this third clinical variant, now referred to as the logopenic variant, in detail. Since that time, it has been confirmed as a distinct clinical phenotype with unique speech and language features. Because this clinical syndrome may be associated with specific pathological and genetic markers, identification and clarification of the features of the logopenic variant may have important ramifications for therapeutic intervention.

\section{Cognitive-linguistic features of the logopenic variant}

Clinical presentation in the logopenic variant is distinct from other PPA subtypes. Recent work has identified spared and impaired cognitive and linguistic processes as well as associated behavioral characteristics.

\section{Speech-language profile}

The initial characterizations of a "logopenic" (from Greek, meaning "lack of words") presentation of PPA described an overall paucity of verbal output, with relative sparing of grammar, phonology, and motor speech [4]. This characterization has been refined and modified in subsequent studies [5,6,7**]. Work by Gorno-Tempini and colleagues [5] identified in the logopenic variant a fluency profile intermediate between those of patients with the semantic and nonfluent variants of PPA. Spoken language was slow in rate, with syntactically simple but accurate utterances and frequent word-finding pauses. Naming was impaired, but single-word comprehension and nonverbal semantic association were relatively spared. Patients had difficulty with comprehension and repetition of sentences, findings which have been interpreted as evidence of phonological working memory impairment [5,6]. With regard to written language processing, individuals with logopenic variant have demonstrated a reading pattern consistent with phonological alexia (selective deficit in pseudoword reading) $\left[7^{* *}, 8\right]$. Spelling impairment has also been noted $\left[9^{*}, 10\right]$, but the precise nature of spelling deficits awaits clarification.

Distinguishing the logopenic variant from the other two major PPA variants has proven challenging, and individuals with this syndrome have likely been grouped with either nonfluent or semantic variant patients in a number of early research reports. Recent work by Wilson and colleagues [11**], however, indicates that several key characteristics of logopenic patients' spontaneous speech can be helpful in differentiating these patients from other PPA syndromes. Based on a speech sample, logopenic patients can be distinguished from nonfluent patients by a lack of speech sound distortions (although phonological paraphasias may be present) and frank syntactic errors. In addition, the maximum speech rate is typically greater in logopenic relative to nonfluent variant patients. The logopenic 
variant can be distinguished from the semantic variant by relatively slower maximum speech rate, presence of phonological paraphasias, and less severe impairment of lexical retrieval (as evidenced by use of lower frequence nouns and fewer pronouns). This work indicates that the logopenic variant of PPA can be differentiated from other PPA variants based on performance on a simple picture description task or other speech sample. (For an example of spontaneous speech during picture description in an individual with logopenic variant, see Video, Supplemental Digital Content 1.) Another study, by Mesulam and colleagues, classified PPA patients into three variants based on a $60 \%$ cutoff for scores on a measure of lexical-semantics (the Peabody Picture Vocabulary Test) and a test of syntax (The

Northwestern Anagram Test) [9*]. Individuals with the logopenic variant scored above $60 \%$ correct on each of these tests, which reliably distinguished them from individuals with nonfluent/agrammatic $(<60 \%$ on the NAT) and semantic ( $<60 \%$ on the PPVT) variants.

\section{Underlying phonological deficit}

Following their initial effort to characterize the logopenic variant as a unique PPA subtype, Gorno-Tempini and colleagues examined six previously unreported logopenic cases in order to further refine the cognitive-linguistic profile of this patient group [6]. In particular, this study aimed to assess the status of the "phonological loop" component of verbal working memory. This aspect of working memory comprises a "store," which holds memory traces for brief periods, as well as a "rehearsal" process that helps to revive traces, which are subject to decay $[12,13]$. Spontaneous speech in this patient group was consistent with previous descriptions, with simple, but grammatically correct utterances and word-finding difficulty. Sentence comprehension deficits, with no effect of syntactic complexity, were again observed, as was a sentence repetition deficit, particularly for low probability sentences. Patients were noted to provide semantically appropriate renditions of repeated sentences (e.g., "The valuable watch was missing" repeated as "The watch was gone"), while failing to repeat targets verbatim, suggesting a semantic, rather than phonological, approach to the task. (For an example of a logopenic patient performing sentence repetition, see Video, Supplemental Digital Content 2.) Several measures were included to assess the integrity of the phonological loop specifically and participants' performance confirmed a deficit in this system. In particular, their span performance for digits and short words was limited to 3, and they were unable to repeat more than a single long word. In addition, patients were only able to repeat series of 3 letters and, unlike normal subjects, showed no benefit of phonological dissimilarity in repetition of letter strings (whereas normal, healthy individuals are better able to repeat sequences of letters whose pronunciations are dissimilar, e.g., C-Y-U vs. T-P-B). Taken together, these results are indicative of phonological loop impairment; however, it remains to be determined whether the disorder affects additional aspects of phonological processing, as detailed assessments of other phonologically demanding tasks (e.g., phoneme manipulation and blending tasks) are lacking in the literature to date.

\section{Associated cognitive and behavioral characteristics}

In addition to the aforementioned speech and language characteristics, several associated cognitive and behavioral characteristics have been identified in the logopenic variant. With regard to neuropsychological profile, individuals with the logopenic variant have been observed to perform worse on tests of calculation than other PPA variants $\left[5,7^{* *}, 14\right]$ and some cases, particularly those with Alzheimer's disease (AD) pathology, have demonstrated impaired performance on memory tasks [15]. Impairment of limb praxis has also been noted [7**]. Studies examining abnormal behavioral characteristics associated with each variant of PPA have identified apathy as a consistent feature in logopenic patients [16,17]. Additiona behavioral features include irritability, anxiety, and agitation [16]. 


\section{Biomarkers in logopenic variant}

Recent research examining biomarkers in the logopenic variant has led to progress in identifying how the clinical presentation relates to underlying anatomical changes as well as pathological and genetic processes.

\section{Imaging Findings}

Volumetric analyses of atrophy in the logopenic variant using voxel-based morphometry (VBM) have identified a pattern of damage primarily affecting the left temporoparietal junction, including the left posterior superior and middle temporal gyri and inferior parietal lobule (see Figure 1) with less consistent involvement of medial temporal and parietal cortex, posterior cingulate, inferior frontal cortex, and contralateral temporo-parietal cortex $\left[5,6,7 * *, 18^{*}\right]$. Some cases show extension of atrophy into inferior and anterior temporal regions and future studies will reveal whether this might be a marker of a specific biological process, such as a genetic mutation. White matter VBM has revealed loss of volume in long association tracts in the left hemisphere [6,7**]. Consistent with findings from structural imaging, an FDG-PET study confirmed a pattern of left temporoparietal hypometabolism in individuals with logopenic variant [19]. The pattern of temporoparietal involvement is similar to that observed in patients with Alzheimer's disease, especially in the early-age-ofonset form [20]. Migliaccio and colleagues [18*] investigated the overlap between the logopenic variant and early-age-of-onset $\mathrm{AD}$ by directly comparing patterns of cortical atrophy in the two clinical syndromes. The results showed a remarkable overlap between the most significantly atrophied regions in posterior temporal and inferior parietal regions with greater involvement of the left temporal cortex in the logopenic variant. Prominent involvement of left temporo-parietal cortex is consistent with the constellation of language and non-language behaviors observed in logopenic variant patients, including phonological deficits, dyscalculia, and limb apraxia, each of which is associated with damage to this region $\left[7^{* *}\right]$.

Molecular imaging techniques have also been applied to the logopenic variant. Rabinovici and colleagues [19] used PET with Pittsburgh compound B (PIB) to investigate the presence of cortical amyloid in patients with PPA in vivo. Consistent with the anatomical studies reported above, logopenic variant patients showed positivity to cortical amyloid similar to patients with $\mathrm{AD}$ in all cases in the small group studied.

\section{Pathological findings}

The prediction that logopenic variant is indicative of underlying AD pathology has been supported not only by observations of temporoparietal atrophy and cortical amyloid binding on PIB-PET, but also by higher than expected occurrence of the apolipoprotein E4 haplotype and CSF biomarkers consistent with AD (elevated tau and reduced Aß42) [5,7**, 18*] (but see [15]). However, pathological confirmation was lacking until Mesulam and colleagues reported that 7/11 consecutive logopenic variant cases had AD pathology at autopsy [15]. Together, these findings provide additional evidence suggesting that the logopenic variant belongs on the spectrum of early onset AD syndromes. In fact, there is a complementary literature examining focal presentations of $\mathrm{AD}$, including language-impaired patients with clinical syndromes that are sometimes, but not always, consistent with the logopenic variant $[21,22,23,24]$. Additional support for $\mathrm{AD}$ as a potential pathological basis in this patient group has come from a retrospective series of PPA patients with AD pathology, in whom a pattern of temporoparietal involvement consistent with the logopenic variant was observed, even when the language syndrome was not explicitly identified as such [25]. More recently, another retrospective study of PPA patients who had pathology or CSF biomarkers consistent with AD identified a logopenic syndrome in all patients $(\mathrm{N}=14)$ 
[26*]. Whereas there is growing evidence for an association between the clinical syndrome and $\mathrm{AD}$ pathology [27], the relationship requires continued investigation via pathologyproven series. It is well known in the field that clinical-pathological associations are probabilistic and not absolute and there are reports of individuals with logopenic variant and non-AD pathology at autopsy $[15,28]$ as well as progranulin mutations that may present with a logopenic-like syndrome (see section on genetic findings, below). In addition, the relation between AD pathology and neural and cognitive changes in the logopenic variant remains to be elucidated, as there does not appear to be a clear correspondence between distribution of pathology (neuritic plaques and neurofibrillary tangles) at autopsy and in-vivo clinical and imaging measures [15].

\section{Genetic findings}

There is growing evidence that progressive aphasic syndromes may, in some cases, have a genetic basis, including mutations of the progranulin (GRN) and microtubule-associated protein tau (MAPT) genes [29,30,30,31,32,33,34,35]. The phenotypes corresponding to each type of mutation have yet to be conclusively identified; however, it appears that GRN mutations may result in a logopenic-like presentation in some cases. In their recent series of nine logopenic patients, Rohrer and colleagues identified two individuals with GRN mutations [ $\left.7^{* *}\right]$. These individuals demonstrated speech-language characteristics and patterns of cortical involvement similar, but not identical to, a subgroup of individuals with logopenic variant and CSF biomarkers consistent with AD. The two individuals with GRN mutations demonstrated, in addition to posterior temporal involvement, damage to the left anterior temporal lobe and, accordingly, exhibited a constellation of language features consistent with semantic impairment. Detailed description of another GRN-mutation case reported, in addition to more classic logopenic variant deficits, grammatical errors, which, in the context of inferior frontal atrophy, suggests possible overlap with the nonfluent variant of PPA [34]. These findings indicate that individuals with GRN mutations may represent a distinct logopenic-like subtype with a partially unique behavioral and anatomical signature that may be of utility in differentiating such cases from those that result from $\mathrm{AD}$ or other pathological processes.

\section{Consensus criteria for diagnosis of the logopenic variant}

An international panel of experts has recently put forth a set of diagnostic criteria for PPA and its clinical variants [36**]. These criteria include both core and supporting features, as well as criteria for imaging and pathology-supported diagnoses. The diagnostic guidelines for logopenic variant include as core features both impaired single-word retrieval in spontaneous speech and impaired repetition of sentences and phrases. Additional supporting features, at least three of which must be present in order to diagnose logopenic variant, include phonological errors in speech, spared single-word comprehension and object knowledge, preservation of motor speech, and lack of agrammatic utterances. In order to reach an imaging-supported diagnosis, the aforementioned clinical features must be accompanied by imaging findings revealing atrophy, hypometabolism, or hypoperfusion of left posterior perisylvian/parietal cortex. Finally, a pathology-confirmed case of logopenic variant requires clinical diagnosis of the syndrome accompanied by histopathological data or the presence of a known genetic mutation. The goal of this work is to provide a standard set of guidelines in order to ensure consistency of diagnosis across clinical and research centers. This will allow for more accurate identification of individuals with the logopenic variant who, ideally, can be followed longitudinally, helping to document the course of illness and, ultimately, via pathology-confirmed series, the underlying pathological substrate(s) in this patient group. 


\section{Conclusion}

The logopenic variant has emerged as a distinct clinical subtype of primary progressive aphasia with a unique cognitive-linguistic, anatomical and, most likely, neuropathological profile. Research to date has clarified the clinical phenotype, suggesting a prominent impairment of phonological memory. Speech and language characteristics, as well as associated non-linguistic deficits are consistent with impairment of temporo-parietal systems critical for language, praxis, and calculation, and imaging studies have confirmed damage to these regions. The first pathology-proven series has identified a potential relationship between logopenic variant and AD pathology. This finding suggests that language phenotype may serve as an important marker for targeting specific disease processes therapeutically. Before this can be accomplished, however, additional and larger series of patients with in vivo biomarker data and pathological confirmation must be collected. In addition, the cognitive-linguistic profile of this patient group requires further examination, particularly in early stages, in order to identify the most reliable diagnostic indicators.

\section{Supplementary Material}

Refer to Web version on PubMed Central for supplementary material.

\section{Acknowledgments}

Funding sources: This work was supported by National Institutes of Health [NIDCD F32 DC010945, NINDS R01 NS050915, NIA P50 AG03006, NIA P01 AG019724]; State of California [DHS 04-35516]; Alzheimer's Disease Research Center of California [03-75271 DHS/ADP/ARCC]; Larry L. Hillblom Foundation; John Douglas French Alzheimer's Foundation; Koret Family Foundation; and McBean Family Foundation.

\section{References}

(1). Mesulam MM. Primary progressive aphasia. Ann.Neurol. 2001; 49(4):425-432. [PubMed: 11310619]

(2). Mesulam MM. Slowly progressive aphasia without generalized dementia. Ann.Neurol. 1982; 11(6):592-598. [PubMed: 7114808]

(3). Grossman M, Ash S. Primary Progressive Aphasia: A Review. Neurocase. 2004; 10(1):3-18. [PubMed: 15849155]

(4). Kertesz A, Davidson W, McCabe P, et al. Primary progressive aphasia: Diagnosis, varieties, evolution. Journal of the International Neuropsychological Society. 2003; 9(05):710-719. [PubMed: 12901777]

(5). Gorno-Tempini ML, Dronkers NF, Rankin KP, et al. Cognition and anatomy in three variants of primary progressive aphasia. Ann.Neurol. 2004; 55(3):335-346. [PubMed: 14991811]

(6). Gorno-Tempini ML, Brambati SM, Ginex V, et al. The logopenic/phonological variant of primary progressive aphasia. Neurology. 2008; 71(16):1227-1234. [PubMed: 18633132]

**(7). Rohrer JD, Ridgway GR, Crutch SJ, et al. Progressive logopenic/phonological aphasia: Erosion of the language network. Neuroimage. 2010; 49(1):984-993. [PubMed: 19679189] This study examined clinical and imaging data in the logopenic variant relative to other PPA variants. It is the first paper to compare findings in individuals with progranulin mutation versus those with likely AD pathology.

(8). Brambati SM, Ogar J, Neuhaus J, et al. Reading disorders in primary progressive aphasia: A behavioral and neuroimaging study. Neuropsychologia. 2009; 47(8-9):1893-1900. [PubMed: 19428421]

*(9). Mesulam M, Wieneke C, Rogalski E, et al. Quantitative template for subtyping primary progressive aphasia. Arch.Neurol. 2009; 66(12):1545-1551. [PubMed: 20008661] This study proposes a novel, quantitative method for subtyping PPA variants using performance on tests of syntax and lexical-semantics. This method identified a unique pattern of sparing of these linguistic abilities in the logopenic variant. 
(10). Sepelyak K, Crinion J, Molitoris J, et al. Patterns of breakdown in spelling in primary progressive aphasia. Cortex. In press.

** (11). Wilson SM, Henry ML, Besbris M, et al. Connected speech production in three variants of primary progressive aphasia. Brain. In press. This study examined connected speech across the three variants of PPA. It is the first to use quantitative measures derived from connected speech to distinguish the logopenic variant from nonfluent and semantic variants.

(12). Baddeley A. Cognitive psychology and human memory. Trends Neurosci. 1988; 11(4):176-181. [PubMed: 2469187]

(13). Baddeley A. Working memory: Looking back and looking forward. Nature Reviews Neuroscience. 2003; 4(10):829-839.

(14). Amici S, Gorno-Tempini ML, Ogar JM, et al. An overview on Primary Progressive Aphasia and its variants. Behavioural Neurology. 2006; 17(2):77-87. [PubMed: 16873918]

(15). Mesulam M, Wicklund A, Johnson N, et al. Alzheimer and frontotemporal pathology in subsets of primary progressive aphasia. Ann.Neurol. 2008; 63(6):709-719. [PubMed: 18412267]

(16). Rohrer JD, Warren JD. Phenomenology and anatomy of abnormal behaviours in primary progressive aphasia. J.Neurol.Sci. 2010; 293(1-2):35-38. [PubMed: 20400120]

(17). Rosen HJ, Allison SC, Ogar JM, et al. Behavioral features in semantic dementia vs other forms of progressive aphasias. Neurology. 2006; 67(10):1752-1756. [PubMed: 17130406]

*(18). Migliaccio R, Agosta F, Rascovsky K, et al. Clinical syndromes associated with posterior atrophy: Early age at onset AD spectrum. Neurology. 2009; 73(19):1571-1578. [PubMed: 19901249] This paper is the first to explore clinical, imaging, and pathologic data in individuals with the logopenic variant of PPA relative to posterior cortical atrophy and early onset Alzhiemer's disease. Findings suggest that the logopenic variant falls within the spectrum of early onset AD syndromes.

(19). Rabinovici GD, Jagust WJ, Furst AJ, et al. A $\beta$ amyloid \& glucose metabolism in three variants of primary progressive aphasia. Ann.Neurol. 2008; 64(4):388-401. [PubMed: 18991338]

(20). Frisoni GB, Pievani M, Testa C, et al. The topography of grey matter involvement in early and late onset Alzheimer's disease. Brain. 2007; (130):720-730. [PubMed: 17293358]

(21). Galton CJ, Patterson K, Xuereb JH, et al. Atypical and typical presentations of Alzheimer's disease: a clinical, neuropsychological, neuroimaging and pathological study of 13 cases. Brain. 2000; 123(3):484-498. [PubMed: 10686172]

(22). Croot K, Hodges JR, Xuereb J, et al. Phonological and Articulatory Impairment in Alzheimer's Disease: A Case Series. Brain Lang. 2000; 75(2):277-309. [PubMed: 11049669]

(23). Alladi S, Xuereb J, Bak T, et al. Focal cortical presentations of Alzheimer's disease. Brain. 2007; 130(10):2636-2645. [PubMed: 17898010]

(24). Stopford CL, Snowden JS, Thompson JC, et al. Variability in cognitive presentation of Alzheimer's disease. Cortex. 2008; 44(2):185-195. [PubMed: 18387548]

(25). Josephs KA, Whitwell JL, Duffy JR, et al. Progressive aphasia secondary to Alzheimer disease vs FTLD pathology. Neurology. 2008; 70(1):25-34. [PubMed: 18166704]

*(26). Rohrer JD, Rossor MN, Warren JD. Alzheimer's pathology in primary progressive aphasia. Neurobiol.Aging. In press. This study reports the largest retrospective series of PPA patients with probable or proven AD pathology since the logopenic variant was fully characterized in the literature.

(27). Deramecourt V, Lebert F, Debachy B, et al. Prediction of pathology in primary progressive language and speech disorders. Neurology. 2010; 74(1):42-49. [PubMed: 19940270]

(28). Grossman M, Xie SX, Libon DJ, et al. Longitudinal decline in autopsy-defined frontotemporal lobar degeneration. Neurology. 2008; 70(22):2036-2045. [PubMed: 18420483]

(29). Krefft TA, Graff-Radford NR, Dickson DW, et al. Familial primary progressive aphasia. Alzheimer Disease \& Associated Disorders. 2003; 17(2):106-112. [PubMed: 12794388]

(30). Snowden JS, Pickering-Brown SM, Mackenzie IR, et al. Progranulin gene mutations associated with frontotemporal dementia and progressive non-fluent aphasia. Brain. 2006; 129(11):30913102. [PubMed: 17003069]

(31). Mesulam M, Johnson N, Krefft TA, et al. Progranulin mutations in primary progressive aphasia: the PPA1 and PPA3 families. Arch.Neurol. 2007; 64(1):43-47. [PubMed: 17210807] 
(32). Snowden JS, Pickering-Brown SM, Du Plessis D, et al. Progressive anomia revisited: Focal degeneration associated with progranulin gene mutation. Neurocase. 2007; 13(5):366-377. [PubMed: 18781435]

(33). Rohrer JD, Warren JD, Barnes J, et al. Mapping the progression of progranulin-associated frontotemporal lobar degeneration. Nature Clinical Practice Neurology. 2008; 4(8):455-460.

(34). Rohrer JD, Crutch SJ, Warrington EK, et al. Progranulin-associated primary progressive aphasia: A distinct phenotype? Neuropsychologia. 2010; 48(1):288-297. [PubMed: 19766663]

(35). van Swieten J, Spillantini MG. Hereditary frontotemporal dementia caused by Tau gene mutations. Brain Pathology. 2007; 17(1):63-73. [PubMed: 17493040]

**(36). Gorno-Tempini ML, Hillis AE, Weintraub S, et al. Recommendations for the classification of primary progressive aphasia and its variants. In press. This paper provides the first comprehensive set of diagnostic criteria for the three variants of PPA, including speech-language features and requirements for imaging and pathology-supported diagnosis. 


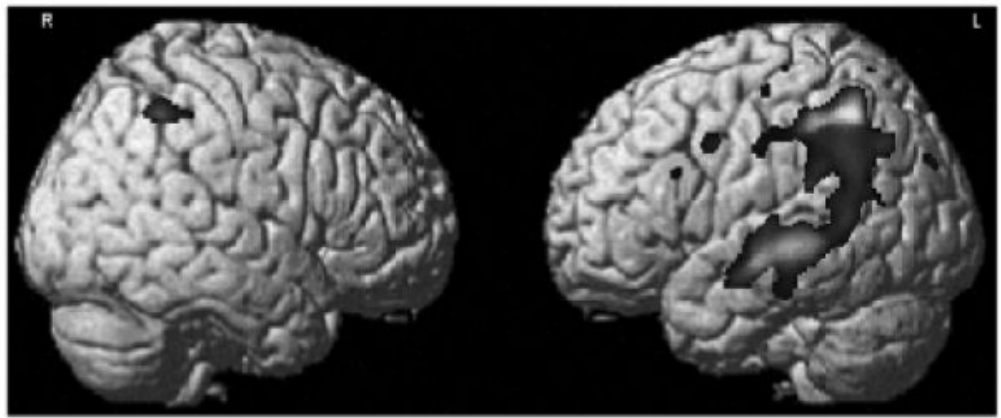

Figure 1. Voxel-based morphometry analysis showing cortical atrophy

Voxel-based morphometry analysis showing cortical atrophy in 10 logopenic variant patients (from Gorno Tempini et al., 2004) compared to 64 normal controls. 